\title{
2-SPANNING CYCLABILITY PROBLEMS OF SOME GENERALIZED PETERSEN GRAPHS
}

\author{
Meng-Chien Yang, LiH-Hsing Hsu \\ Department of Computer Science and Communication Engineering \\ Providence University, Taichung, Taiwan 43301, R.O.C. \\ e-mail: mcyang2@pu.edu.tw \\ lihhsing@gmail.com \\ Chun-NAn Hung \\ Department of Information Management, Da-Yeh University \\ No.168, University Rd., Dacun, Changhua, Taiwan 51591, R.O.C. \\ e-mail: spring@mail.dyu.edu.tw
}

AND

\section{EDdie Cheng}

Department of Mathematics and Statistics Oakland University, Rochester, MI 48309

e-mail: echeng@oakland.edu

\begin{abstract}
A graph $G$ is called $r$-spanning cyclable if for every $r$ distinct vertices $v_{1}, v_{2}, \ldots, v_{r}$ of $G$, there exists $r$ cycles $C_{1}, C_{2}, \ldots, C_{r}$ in $G$ such that $v_{i}$ is on $C_{i}$ for every $i$, and every vertex of $G$ is on exactly one cycle $C_{i}$. In this paper, we consider the 2-spanning cyclable problem for the generalized Petersen graph $G P(n, k)$. We solved the problem for $k \leq 4$. In addition, we provide an additional observation for general $k$ as well as stating a conjecture.
\end{abstract}

Keywords: Petersen graph, spanning cyclable.

2010 Mathematics Subject Classification: 05C38, 05C45.

\section{REFERENCES}

[1] M. Albert, R.E.L. Aldred and D. Holton, On $3^{*}$-connected graphs, Australas. J. Combin. 24 (2001) 193-207. 
[2] B. Alspach, The classification of Hamiltonian generalized Petersen graphs, J. Combin. Theory Ser. B 34 (1983) 293-312.

doi:10.1016/0095-8956(83)90042-4

[3] B. Alspach, D. Bryant and D. Dyer, Paley graphs have Hamilton decompositions, Discrete Math. 312 (2012) 113-118.

doi:10.1016/j.disc.2011.06.003

[4] B. Alspach and J. Liu, On the Hamilton connectivity of generalized Petersen graphs, Discrete Math. 309 (2009) 5461-5473. doi:10.1016/j.disc.2008.12.016

[5] M. Behzad, P. Hatami and E.S. Mahmoodian, Minimum vertex covers in the generalized Petersen graphs $P(n, 2)$, Bull. Inst. Combin. Appl. 56 (2009) 98-102.

[6] J.A. Bondy, Pancyclic graphs I, J. Combin. Theory Ser. B 11 (1971) 80-84. doi:10.1016/0095-8956(71)90016-5

[7] J.A. Bondy, Variations on the Hamiltonian theme, Canad. Math. Bull. 15 (1972) $57-62$. doi:10.4153/CMB-1972-012-3

[8] M.Y. Chan and S.J. Lee, On the existence of Hamiltonian circuits in faulty hypercubes, SIAM J. Discrete Math. 4 (1991) 511-527. doi: $10.1137 / 0404045$

[9] R.J. Faundree, Survey of results on k-ordered graphs, Discrete Math. 229 (2001) 73-87. doi:10.1016/S0012-365X(00)00202-8

[10] J.R. Faundree, R.J. Gould, A.V. Kostochka, L. Lesniak, I. Schiermeyer and A. Saito, Degree conditions for k-ordered Hamiltonian graphs, J. Graph Theory 42 (2003) 199-210. doi:10.1002/jgt.10084

[11] S. Fujita and T. Araki, Three-round adaptive diagnosis in binary $n$-cubes, Lecture Notes in Comput. Sci. 3341 (2004) 442-451. doi:10.1007/978-3-540-30551-4_39

[12] S.L. Hakimi and E.F. Schmeichel, On the number of cycles of length $k$ in a maximal planar graph, J. Graph Theory 3 (1979) 69-86. doi:10.1002/jgt.3190030108

[13] C.-N. Hung, D. Lu, R. Jia, C.-K. Lin, L. Lipták, E. Cheng, J.J.M. Tan and L.-H. Hsu, 4-ordered Hamiltonian problems for the generalized Petersen graph $\operatorname{GP}(n, 4)$, Math. Comput. Modelling 57 (2013) 595-601. doi:10.1016/j.mcm.2012.07.022

[14] S.Y. Hsieh, G.H. Chen and C.W. Ho, Fault-free Hamiltonian cycles in faulty arrangement graphs, IEEE Trans. Parallel Distributed Systems 10 (1999) 223-237. doi:10.1109/71.755822

[15] L.-H. Hsu and C.-K. Lin, Graph Theory and Interconnection Networks (CRC Press, 2009). 
[16] L.-H. Hsu, J.M. Tan, E. Cheng, L. Lipták, C.K. Lin and M. Tsai, Solution to an open problem of 4-ordered Hamiltonian graphs, Discrete Math. 312 (2012) 23562370 .

doi:10.1016/j.disc.2012.04.003

[17] M. Lewinter and W. Widulski, Hyper-Hamilton laceable and caterpillar-spannable product graphs, Comput. Math. Appl. 34 (1997) 99-104. doi:10.1016/S0898-1221(97)00223-X

[18] R. Li, S. Li and Y. Guo, Degree conditions on distance 2 vertices that imply $k$-ordered Hamiltonian, Discrete Appl. Math. 158 (2010) 331-339. doi:10.1016/j.dam.2009.05.005

[19] C.-K. Lin, H.-M. Huang and L.-H. Hsu, The super connectivity of the pancake graphs and super laceability of the star graphs, Theoret. Comput. Sci. 339 (2005) 257-271. doi:10.1016/j.tcs.2005.02.007

[20] C.-K. Lin, H.-M. Huang, J.J.M. Tan and L.-H. Hsu, On spanning connected graphs, Discrete Math. 308 (2008) 1330-1333. doi:10.1016/j.disc.2007.03.072

[21] J. Liu, Hamiltonian decompositions of Cayley graphs on Abelian groups, Discrete Math. 131 (1994) 163-171. doi:10.1016/0012-365X(94)90381-6

[22] J. Liu, Hamiltonian decompositions of Cayley graphs on abelian groups of even order, J. Combin. Theory Ser. B 88 (2003) 305-321. doi:10.1016/S0095-8956(03)00033-9

[23] K. Mészáros, On 3-regular 4-ordered graphs, Discrete Math. 308 (2008) 2149-2155. doi:10.1016/j.disc.2007.04.061

[24] L. Ng and M. Schultz, k-ordered Hamiltonian graphs, J. Graph Theory 24 (1997) 45-57. doi:10.1002/(SICI)1097-0118(199701)24:1〈45::AID-JGT6〉3.0.CO;2-J

[25] G.N. Robertson, Graphs Minimal under Girth, Valency and Connectivity Constraints, PhD Thesis (University of Waterloo, 1968).

[26] D.R. Silaban, A. Parestu, B.N. Herawati, K.A. Sugeng and Slamin, Vertex-magic total labelings of union of generalized Petersen graphs and union of special circulant graphs, J. Combin. Math. Combin. Comput. 71 (2009) 201-207.

[27] C. Tong, X. Lin, Y. Yang and M. Luo, 2-rainbow domination of generalized Petersen graphs $P(n, 2)$, Discrete Appl. Math. 157 (2009) 1932-1937. doi:10.1016/j.dam.2009.01.020

[28] M.E. Watkins, A theorem on Tait colorings with an application to the generalized Petersen graphs, J. Combin. Theory 6 (1969) 152-164. doi:10.1016/S0021-9800(69)80116-X

[29] G. Xu, 2-rainbow domination in generalized Petersen graphs $P(n, 3)$, Discrete Appl. Math. 157 (2009) 2570-2573. doi:10.1016/j.dam.2009.03.016 
Received 18 September 2017

Revised 5 March 2018

Accepted 5 March 2018 